remaining 58, 31 showed edema and LGE typical of AM and 9 had non-ischemic LGE suggesting possible myocarditis. 9 patients had acute infarcts and 9 were normal. Seven of the normal patients had troponin I rise of $<2 \mu \mathrm{g} / \mathrm{L}$.

Conclusion Suspected acute myocarditis is a common indication for CMR. None of our cohort had a positive CMR for AM if troponin I was $<0.5 \mu \mathrm{g} / \mathrm{L}$. The demographics of positive cases mirrors previous series in terms of age and gender. Interestingly, the majority of positive AM cases had normal biventricular function (26/40 or 65\%). Longterm outcome of these patients has yet to be established and routine follow-up CMR may not be needed.

\section{CALCIUM SCORES IN SYMPTOMATIC PATIENTS UNDERGOING CT CORONARY ANGIOGRAMS: IS THERE ANY VALUE?}

Julia Sun, Yashoda Gurung Koney, Prem Ruben Jayaram, Mary-Jane Bennie, Hilmar Spohr, John Curtin. Norfolk and Norwich University Hospital, UK

\subsection{6/heartjnl-2019-BSCI.14}

Introduction NICE guideline 'chest pain of recent onset: assessment and diagnosis' (CG95) recommends CT Coronary Angiography (CTCA) as the first line investigation for stable chest pain if clinically indicated. The aim is to investigate the need for a coronary artery calcium (CAC) score before CTCA.

Methods Retrospective data was collected on patients who underwent both CAC and CTCA from 2016 to 2018. Patients were excluded if they had any previous intervention. Population characteristics and outcomes by year were analysed with Chi-Squared test. Clinically relevant findings were defined as $>50 \%$ coronary artery stenosis or malignant aberrant courses. Relationship between age and calcium score was analysed with Spearma's Rank, regression analysis and receiver operating characteristics (ROC).

Results 1665 patients were identified and 164 were excluded, leaving 1501 patients $(\mathrm{F}=735, \quad \mathrm{M}=766$; Range=17-94; Median =58). The proportion of clinically relevant findings were not significantly changed over the period of study $(\mathrm{p}>0.2)$. The proportion of normal scans has decreased from $61 \%$ in 2016 to $46 \%$ in 2018, with a corresponding increase in mild artery stenosis $(\mathrm{p}<0.0001)$. There is positive correlation with calcium score and age $(r=0.33, p<0.0001)$. Regression analysis of calcium scores shows linear regression with age $\left(\mathrm{R}^{2}=0.09\right)$; the cohort under 42 years had negligible calcium scores. ROC analysis shows age is predictive of calcium scores $>800\left(\mathrm{AUC}_{\text {age: } \text { male }}=0.79 ; \mathrm{AUC}_{\text {age: female }}=0.88\right)$

Conclusion There was an increase in proportion of patients with mild disease but no significant change in those with clinically relevant findings. Performing a CAC prior to CTCA for patients under 42 years old provides poor value.

\section{DIAGNOSTIC UTILITY AND SAFETY OF CORONARY CT ANGIOGRAPHY IN PRE-RENAL TRANSPLANT PATIENTS}

\footnotetext{
${ }^{1}$ Victor Voon, ${ }^{1}$ Nanci $O^{\prime}$ Reilly, ${ }^{2}$ Gerlineke Hawkins-VanDerCingel, ${ }^{1}$ Francesca Pugliese, ${ }^{1}$ Emma Cheasty, ${ }^{2}$ Neil Ashman, ${ }^{1}$ Ceri Davies. 'Barts Heart Centre, Barts Health NHS Trust, St Bartholomew's Hospital, London, UK; ${ }^{2}$ Nephrology Department, Royal London Hospital, London, UK
}

10.1136/heartjnl-2019-BSCl.15
Background Emerging evidence suggests a potential role of coronary computed tomography angiography (CCTA) for coronary assessment pre-renal transplantation. Therefore, we aimed to evaluate the diagnostic utility and safety of CCTA in such patients.

Methods We retrospectively evaluated data from 58 consecutive patients who had pre-renal transplant CCTA between 2010-2018. The diagnostic value of non-obstructive $(<70 \%$ stenosis) and obstructive ( $\geq 70 \%$ stenosis) coronary artery disease by CCTA in predicting subsequent myocardial infarction (MI) and/or percutaneous coronary intervention (PCI) was assessed. Results were expressed as mean \pm SD.

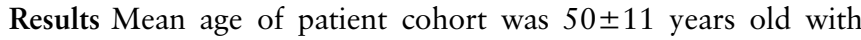
a follow-up duration of $46 \pm 20$ months from CCTA. Baseline demographics include male (58\%), hypertension (65\%), diabetes (42\%), hemodialysis (70\%), peritoneal dialysis (18\%), not on dialysis (12\%). Among those not on dialysis, no patients experienced contrast-induced nephropathy post-CCTA. All patients subsequently underwent renal transplant. CCTA demonstrated mean DLP $503 \pm 535 \mathrm{mGym}^{2}$ and calcium score 167 \pm 309 . Number of patients with obstructive coronary disease: 1 -vessel $(n=5), 2$-vessels $(n=6), 3$-vessels $(n=1)$. Independent of symptoms, CCTA demonstrated a positive predictive value $41 \%$, negative predictive value $100 \%$, sensitivity $100 \%$, and specificity $86 \%$, in predicting subsequent $\mathrm{MI} / \mathrm{PCI}$ over the follow-up period.

Conclusion In this cohort of pre-renal transplant patients, CCTA is safe, and has a high sensitivity and negative predictive value in ruling out obstructive coronary disease and subsequent $\mathrm{MI} / \mathrm{PCI}$ over a 4-year follow-up period. CCTA also acts as a valuable diagnostic gatekeeper prior to subsequent functional and/or invasive testing.

\section{EFFECT OF A CALCIUM DEBLOOMING ALGORITHM ON THE ACCURACY OF CORONARY COMPUTED TOMOGRAPHY ANGIOGRAPHY}

${ }^{1}$ Jonathan R Weir-McCall, ${ }^{2}$ Rui Wang, ${ }^{3}$ Jaydeep Halankar, ${ }^{4}$ Jiang Hsieh, ${ }^{3}$ Cameron Hague, ${ }^{3}$ Samuel Rosenblatt, ${ }^{2}$ Zhanming Fan, ${ }^{2}$ Lei $\mathrm{Xu}^{*},{ }^{3}$ Jonathon A Leipsic. 'Department of Radiology, University of Cambridge, Cambridge; ${ }^{2}$ Department of Radiology, Beijing Anzhen Hospital, Capital Medical University, Beijing; ${ }^{3}$ St Pauls Hospital, Vancouver; ${ }^{4} G E$ Healthcare Technologies, Waukesha, Wisconsin

\subsection{6/heartjnl-2019-BSCI.16}

Introduction Coronary artery calcification is a significant contributor to reduced accuracy of coronary computed tomographic angiography (CTA) in the assessment of coronary artery disease severity. The aim of the current study is to assess the impact of a prototype calcium deblooming algorithm on the diagnostic accuracy of CTA.

Methods 40 patients referred for invasive catheter angiography underwent CTA and invasive catheter angiography. CTA studies were read with and without the deblooming algorithm blinded to the invasive coronary angiogram findings. Sensitivity, specificity, accuracy, positive predictive value and negative predictive value for the detection of stenosis $\geq 50 \%$ were evaluated using quantitative coronary angiography as the reference standard.

Results All studies were diagnostic with 581 segments available for evaluation. Image score was $3.64 \pm 0.72$ with $\mathrm{CTA}_{\mathrm{DE}}$ BLOOM, versus $3.56 \pm 0.72$ with CTASTAND $(p=0.38)$. CTADEBLOOM had significantly less calcium blooming artifact than CTASTAND $(12.5 \%$ vs. $47.5 \%, \quad \mathrm{p}=0.001)$. The 